\title{
UNITARY ASYMPTOTES OF HILBERT SPACE OPERATORS
}

\author{
LÁSZLÓ KÉRCHY \\ Bolyai Institute, Attila József University \\ Aradi vértanúk tere 1, 6720 Szeged, Hungary
}

In this survey article we are going to present the effectiveness of the use of unitary asymptotes in the study of Hilbert space operators.

1. Power bounded operators. In operator theory it is a generally used, fruitful method that in order to explore the structure and properties of operators belonging to a large, undetected class one relates these operators to those of a special, well-understood class, and then exploiting this connection obtains theorems on the operators in the large class. Unitary operators, the automorphisms of Hilbert spaces form certainly the most thoroughly investigated and best understood class. It is sufficient to refer to the spectral theorem which is one of the main tools in their study (see e.g. [29], [30] or [6]). On the other hand, power bounded operators, that is, the operators with bounded sequence of iterates form an extensive, broad class. The idea of using Banach limits to relate power bounded operators to unitaries stems from the paper [33] by B. Sz.-Nagy. It was shown in that paper that every invertible power bounded operator with a power bounded inverse is similar to a unitary operator. It was observed in [22] that Sz.-Nagy's method works for every power bounded operator and that the unitary operator associated with the power bounded operator has a useful property of universality.

To be more precise, let us give the exact definitions. Let $\mathcal{H}$ be a complex Hilbert space and let $\mathcal{B}(\mathcal{H})$ denote the set of all bounded linear operators acting on $\mathcal{H}$. Consider a power bounded operator $T \in \mathcal{B}(\mathcal{H})$, that is, $\sup \left\{\left\|T^{n}\right\|: n=\right.$ $0,1,2, \ldots\}$ is finite. Let $L$ be a Banach limit on the sequence space $\ell^{\infty}$, that is, let $L$ be a positive linear functional with the properties $L(1,1,1, \ldots)=1$ and $L\left(\left\{c_{n}\right\}_{n=0}^{\infty}\right)=L\left(\left\{c_{n+1}\right\}_{n=0}^{\infty}\right)$. The existence of such an $L$, which is an extension

1991 Mathematics Subject Classification: 47A20, 47A45, 47A15, 47A10.

The paper is in final form and no version of it will be published elsewhere. 
of the concept of limit from convergent sequences to all bounded sequences, was proved by S. Banach (see [1] and [6]). Denoting by $\langle\cdot, \cdot\rangle$ the original inner product on $\mathcal{H}$ we can introduce a new semi-inner product by the formula

$$
\langle x, y\rangle_{T}:=L\left(\left\{\left\langle T^{n} x, T^{n} y\right\rangle\right\}_{n=0}^{\infty}\right) \quad \text { for } x, y \in \mathcal{H} .
$$

Factorization and completion yield a Hilbert space $\mathcal{H}_{+, T}^{(\mathrm{a})}$, and the operator $T$ induces an isometry $T_{+}^{(\mathrm{a})}$ on $\mathcal{H}_{+, T}^{(\mathrm{a})}$. The natural embedding $X_{+, T}$ of $\mathcal{H}$ into $\mathcal{H}_{+, T}^{(\mathrm{a})}$ is a bounded linear transformation which intertwines the operators $T$ and $T_{+}^{(a)}$ : $X_{+, T} T=T_{+}^{(\mathrm{a})} X_{+, T}$. Let $T^{(\mathrm{a})}$ be the minimal unitary extension of the isometry $T_{+}^{(\mathrm{a})}$ acting on the space $\mathcal{H}_{T}^{(\mathrm{a})}=\bigvee_{n=0}^{\infty}\left(T^{(\mathrm{a})}\right)^{-n} \mathcal{H}_{+, T}^{(\mathrm{a})}$. Then $X_{T} T=T^{(\mathrm{a})} X_{T}$ where the transformation $X_{T} \in \mathcal{B}\left(\mathcal{H}, \mathcal{H}_{T}^{(\mathrm{a})}\right)$ is defined by $X_{T} h:=X_{+, T} h(h \in \mathcal{H})$. We shall also use the notation

$$
X_{T} \in \mathcal{I}\left(T, T^{(\mathrm{a})}\right):=\left\{Q \in \mathcal{B}\left(\mathcal{H}, \mathcal{H}^{(\mathrm{a})}\right): Q T=T^{(\mathrm{a})} Q\right\} .
$$

The operators $T_{+}^{(\mathrm{a})}$ and $T^{(\mathrm{a})}$ are called, respectively, the isometric and unitary asymptotes of the power bounded operator $T$.

It turns out that the unitary asymptote $T^{(\mathrm{a})}$ together with the canonical intertwining transformation $X_{T}$ are universal in a natural sense and that this property determines the pair $\left(X_{T}, T^{(\mathrm{a})}\right)$. This is the content of the following theorem whose proof can be found in [22].

THEOREM 1. (a) Every bounded linear transformation A intertwining the power bounded operator $T$ with a unitary operator $U$ can be uniquely factored as $A=B X_{T}$, where the bounded linear transformation $B$ intertwines the operators $T^{(\mathrm{a})}$ and $U$.

(b) Furthermore, if $W$ is a unitary operator and $Z \in \mathcal{I}(T, W)$ is a transformation such that the pair $(Z, W)$ has the above property of universality, then there exists an invertible mapping $S \in \mathcal{I}\left(T^{(\mathrm{a})}, W\right)$ satisfying $Z=S X_{T}$. We say that the pair $(Z, W)$ is similar to $\left(X_{T}, T^{(\mathrm{a})}\right)$.

An analogous statement holds for the isometric asymptote $T_{+}^{(a)}$ and the canonical intertwining transformation $X_{+, T}$. We remark that, as an immediate consequence of Theorem 1, up to similarity the pair $\left(X_{T}, T^{(\mathrm{a})}\right)$ does not depend on the choice of the Banach limit $L$.

It is frequently important to know how the unitary asymptote $T^{(\mathrm{a})}$ can be computed from the matrix of the operator $T$ taken with respect to a (finite) decomposition of the space $\mathcal{H}$. By the following theorem of [22], if the matrix is triangular then $T^{(\mathrm{a})}$ is the orthogonal sum of the unitary asymptotes of the diagonal entries.

THEOREM 2. Let $\mathcal{M}$ be an invariant subspace of the power bounded operator $T \in \mathcal{B}(\mathcal{H})$. Let $T_{1}$ denote the restriction of $T$ to $\mathcal{M}$ and let $T_{2}$ be the compression of $T$ to $\mathcal{N}=\mathcal{H} \ominus \mathcal{M}$, in notation $T_{1}=T \mid \mathcal{M}$ and $T_{2}=P_{\mathcal{N}} T \mid \mathcal{N}$, where $P_{\mathcal{N}}$ stands 
for the orthogonal projection onto $\mathcal{N}$. Then the unitary asymptote $T^{(\mathrm{a})}$ is unitarily equivalent to the orthogonal sum $T_{1}^{(\mathrm{a})} \oplus T_{2}^{(\mathrm{a})}$.

The closer the connection between the power bounded operator $T$ and its unitary asymptote $T^{(\mathrm{a})}$, the more information can be expected from the study of $T^{(\mathrm{a})}$. The canonical intertwining operator $X_{T}$ is one-to-one (a good behaviour) if and only if $\inf \left\{\left\|T^{n} x\right\|: n=0,1,2, \ldots\right\}>0$ for every nonzero $x \in \mathcal{H}$. In that case we say that $T$ is of class $C_{1}$. If, on the contrary, $\inf \left\{\left\|T^{n} x\right\|: n=0,1,2, \ldots\right\}=0$ for every $x \in \mathcal{H}$, then $T$ is said to be of class $C_{0}$., the Hilbert space $\mathcal{H}_{T}^{(\mathrm{a})}$ reduces to the zero space and no information can be derived from $T^{(\mathrm{a})}$. If the adjoint $T^{*}$ of $T$ is of class $C_{\beta}$. then we say that $T$ is of class $C_{\cdot \beta}(\beta=0,1)$, and for $\alpha, \beta=0,1$ the class $C_{\alpha \beta}$ is defined to be the intersection $C_{\alpha}$. $\cap C_{. \beta}$.

Suppose now that the power bounded operator $T$ belongs to the class $C_{11}$, which is the best case from our point of view. Since $\left(X_{+, T}\right)^{*} \in \mathcal{I}\left(\left(T_{+}^{(\mathrm{a})}\right)^{*}, T^{*}\right)$ is one-to-one, the assumption $T \in C_{.1}$ implies that $\left(T_{+}^{(a)}\right)^{*}$ is unitary. Thus $X_{T}$ is a quasi-affinity, which means that $X_{T}$ is an injective transformation with dense range. Since the system $\mathcal{I}\left(T, T^{(\mathrm{a})}\right)$ of intertwining mappings contains a quasiaffinity, $T$ is a quasi-affine transform of its unitary asymptote $T^{(a)}$, in notation: $T \prec T^{(\text {a) }}$. Repeating the previous argument for $T^{*}$, and using the assumption $T \in C_{1}$, we infer that $T_{*}^{(\mathrm{a})}:=\left(\left(T^{*}\right)^{(\mathrm{a})}\right)^{*} \prec T$, hence $T_{*}^{(\mathrm{a})} \prec T^{(\mathrm{a})}$. Since $T_{*}^{(\mathrm{a})}$ and $T^{(\mathrm{a})}$ are unitary, a short technical manipulation with the polar decomposition of the intertwining quasi-affinity shows that $T_{*}^{(\mathrm{a})}$ and $T^{(\mathrm{a})}$ are unitarily equivalent. Therefore, both $T \prec T^{(\mathrm{a})}$ and $T^{(\mathrm{a})} \prec T$, and in that case we say that $T$ and $T^{(\mathrm{a})}$ are quasi-similar, in notation $T \sim T^{(\mathrm{a})}$. The concept of quasi-similarity was introduced by B. Sz.-Nagy and C. Foiaş, and proved to be an effective tool in creating canonical models in different classes of operators. (See e.g. [35] and [2].)

Exploiting the quasi-similarity of $T$ and $T^{(\mathrm{a})}$ we can conclude that $T$ has an abundance of invariant subspaces, in fact, the invariant subspace lattice Lat $T$ of $T$ has a subsystem which is isomorphic to the lattice of spectral subspaces of $T^{(\mathrm{a})}$.

To be more precise, let $\{T\}^{\prime}:=\mathcal{I}(T, T)$ denote the commutant of $T$, and let Hyplat $T$ stand for the lattice of hyperinvariant subspaces, that is,

$$
\text { Hyplat } T:=\bigcap\left\{\text { Lat } Q: Q \in\{T\}^{\prime}\right\} .
$$

Let Hyplat ${ }_{1} T$ be the subset of Hyplat $T$ consisting of quasi-reducing subspaces, that is,

$$
\text { Hyplat }_{1} T:=\left\{\mathcal{M} \in \operatorname{Hyplat} T: T \mid \mathcal{M} \text { is of class } C_{11}\right\} .
$$

It is clear that Hyplat $T^{(\mathrm{a})}=$ Hyplat $_{1} T^{(\mathrm{a})}$ and if $\mathcal{H}$ is separable then Hyplat $T^{(\mathrm{a})}$ coincides with the set of spectral subspaces.

Consider an arbitrary operator $A$ in the commutant $\{T\}^{\prime}$ of $T$. Since $X_{T} A \in$ $\mathcal{I}\left(T, T^{(\mathrm{a})}\right)$, by Theorem 1 there exists a unique operator $B \in\left\{T^{(\mathrm{a})}\right\}^{\prime}$ such that $X_{T} A=B X_{T}$. It is easy to verify that the mapping

$$
\gamma_{T}:\{T\}^{\prime} \rightarrow\left\{T^{(\mathrm{a})}\right\}^{\prime}, \quad A \mapsto B,
$$


is an algebra homomorphism such that $\gamma_{T}(I)=I$ and $\gamma_{T}(T)=T^{(\mathrm{a})}$. Applying these considerations to the adjoint of $T$ and introducing the notation $Y_{T}:=$ $\left(X_{T^{*}}\right)^{*}$ we infer that for every operator $C \in\{T\}^{\prime}$ there exists a unique $D \in\left\{T_{*}^{(\mathrm{a})}\right\}^{\prime}$ such that $C Y_{T}=Y_{T} D$. Furthermore, the mapping

$$
\widetilde{\gamma}_{T}:\{T\}^{\prime} \rightarrow\left\{T_{*}^{(\mathrm{a})}\right\}^{\prime}, \quad C \mapsto D,
$$

is an algebra homomorphism, $\widetilde{\gamma}_{T}(I)=I$ and $\widetilde{\gamma}_{T}(T)=T_{*}^{(\mathrm{a})}$. Now, it is easy to see that for every hyperinvariant subspace $\mathcal{N}$ for $T_{*}^{(\mathrm{a})}$ the subspace $\left(Y_{T} \mathcal{N}\right)^{-}$is quasi-reducing and hyperinvariant for $T$. It can, furthermore, be proved (see [22]) that the mapping obtained in this way implements an isomorphism between the lattices Hyplat $T_{*}^{(\mathrm{a})}$ and Hyplat ${ }_{1} T$.

THEOREM 3. If $T$ is a power bounded operator of class $C_{11}$, then the mapping

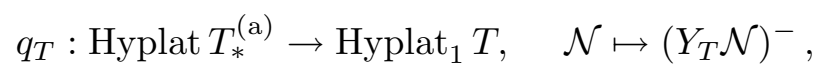

is the unique lattice isomorphism such that $T_{*}^{(\mathrm{a})} \mid \mathcal{N}$ is quasi-similar to $T \mid q_{T}(\mathcal{N})$ for every subspace $\mathcal{N} \in$ Hyplat $T_{*}^{(\mathrm{a})}$.

We note that these investigations have been extended in [3] by $\mathrm{H}$. Bercovici to $n$-tuples of commuting power bounded operators, and even more: to (uniformly) bounded representations of an arbitrary commutative semigroup $S$. Here the role of the Banach limit is taken over by an invariant mean $\phi$ on $S$, which is, by definition, a translation invariant positive linear functional of norm 1 on the space $\ell^{\infty}(S)$. The existence of such a $\phi$ was proved by M. Day (see [7] and [13]).

2. Contractions. Let us now restrict our attention to Hilbert space contractions. So let $T$ be a contraction acting on the Hilbert space $\mathcal{H}$, that is, $T \in \mathcal{B}(\mathcal{H})$ and $\|T\| \leq 1$. Sz.-Nagy's celebrated dilation theorem on the existence of a unitary power dilation and the functional model elaborated by Sz.-Nagy and Foias provide us with powerful tools in the study of $T$. (See [34], [27] and [35].) Let us examine how the unitary asymptote $T^{(\mathrm{a})}$ can be fitted into this theory.

By the dilation theorem there exists an essentially unique unitary operator $U_{T}$ on a larger space $\mathcal{K}_{T}$ such that $\left\langle U_{T}^{n} x, y\right\rangle=\left\langle T^{n} x, y\right\rangle$ for every $x, y \in \mathcal{H}$ and $n=0,1,2, \ldots$, and the smallest reducing subspace of $U_{T}$ containing $\mathcal{H}$ is $\mathcal{K}_{T}$. The operator $U_{T}$ is called the minimal unitary dilation of the contraction $T$.

It is easy to verify that the subspace $\mathcal{L}_{T}=\left(\left(U_{T}-T\right) \mathcal{H}\right)^{-}$is wandering for $U_{T}$, that is, $U_{T}^{n} \mathcal{L}_{T}$ is orthogonal to $\mathcal{L}_{T}$, for every positive integer $n$. The orthogonal sum $\mathcal{M}\left(\mathcal{L}_{T}\right):=\sum_{n=-\infty}^{\infty} \oplus U_{T}^{n} \mathcal{L}_{T}$ reduces $U_{T}$ to a bilateral shift of multiplicity $\operatorname{dim} \mathcal{L}_{T}$. Consider the orthogonal complement $\mathcal{R}_{*, T}:=\mathcal{K}_{T} \ominus \mathcal{M}\left(\mathcal{L}_{T}\right)$. The unitary operator $R_{*, T}:=U_{T} \mid \mathcal{R}_{*, T}$ is called the $*$-residual part of $U_{T}$.

It is plain that the subspace $\mathcal{M}_{+}\left(\mathcal{L}_{T}\right):=\sum_{n=0}^{\infty} \oplus U_{T}^{n} \mathcal{L}_{T}$ is orthogonal to $\mathcal{H}$ and invariant for $U_{T}$. Hence the subspace $\mathcal{K}_{T-}:=\mathcal{K}_{T} \ominus \mathcal{M}_{+}\left(\mathcal{L}_{T}\right)$ contains $\mathcal{H}$, it is invariant for $U_{T}^{*}$ and $\mathcal{K}_{T-}=\mathcal{R}_{*, T} \oplus \mathcal{M}_{-}\left(\mathcal{L}_{T}\right)$ provides the Wold decomposition of the isometry $U_{T}^{*} \mid \mathcal{K}_{T-}$, where $\mathcal{M}_{-}\left(\mathcal{L}_{T}\right)=\mathcal{M}\left(\mathcal{L}_{T}\right) \ominus \mathcal{M}_{+}\left(\mathcal{L}_{T}\right)$. Since 
$\left(U_{T}^{*} \mid \mathcal{K}_{T-}\right)^{*} \mid \mathcal{H}=T$ we infer that, for every $h \in \mathcal{H}$, the sequence $\left\{U_{T}^{-n} T^{n} h\right\}_{n=0}^{\infty}$ strongly converges to a vector $\widehat{X}_{T} h$, which is the orthogonal projection of $h$ onto the subspace $\mathcal{R}_{*, T}$. It readily follows that $\widehat{X}_{T} \in \mathcal{I}\left(T, R_{*, T}\right)$ and $\left\|\widehat{X}_{T} h\right\|=$ $\lim _{n \rightarrow \infty}\left\|T^{n} h\right\|$ for every $h \in \mathcal{H}$. Applying Theorem 1 we conclude that the $*-$ residual part $R_{*, T}$ of $U_{T}$ corresponds to the unitary asymptote $T^{(\mathrm{a})}$ of $T$ in the dilation space.

THEOREM 4. For every Hilbert space contraction $T$, the pair $\left(X_{T}, T^{(\mathrm{a})}\right)$ is equivalent to the pair $\left(\widehat{X}_{T}, R_{*, T}\right)$, that is, there exists a unitary transformation $Z \in \mathcal{I}\left(R_{*, T}, T^{(\mathrm{a})}\right)$ such that $X_{T}=Z \widehat{X}_{T}$.

A more detailed examination of the geometric structure of the dilation space led to the construction of the functional model for T. (See [35] and [25].) First of all, suppose that the contraction $T$ is completely nonunitary (c.n.u.), that is, $T$ has no nontrivial unitary summand. The defect operator of $T$ is, by definition, the positive operator $D_{T}:=\left(I-T^{*} T\right)^{1 / 2}$. It is clear that $\|T h\|^{2}=\|h\|^{2}-\left\|D_{T} h\right\|^{2}$ for every $h \in \mathcal{H}$, and that $\operatorname{ker} D_{T}$ is the subspace consisting of those vectors whose norms do not alter under $T$. The defect space $\mathcal{D}_{T}$ of $T$ is the orthogonal complement $\mathcal{D}_{T}:=\left(\operatorname{ran} D_{T}\right)^{-}$. The contractive operator-valued, analytic function $\Theta_{T}$ defined on the open unit disc $\mathbb{D}$ of the complex plane $\mathbb{C}$ by the formula

$$
\Theta_{T}: \mathbb{D} \rightarrow \mathcal{B}\left(\mathcal{D}_{T}, \mathcal{D}_{T^{*}}\right), \quad \Theta_{T}(z):=\left(-T+z D_{T^{*}}\left(I-z T^{*}\right)^{-1} D_{T}\right) \mid \mathcal{D}_{T},
$$

is called the characteristic function of the contraction $T$.

The characteristic function $\Theta_{T}$ is purely contractive, that is, $\|\Theta(0) x\|<\|x\|$ for every non-zero vector $x \in \mathcal{D}_{T}$, and has nontangential limit in the strong operator topology almost everywhere (a.e. on the unit circle $\partial \mathbb{D}$. This contractive, measurable limit function defined a.e. on $\partial \mathbb{D}$ will also be denoted by $\Theta_{T}$. Let $\Delta_{T}: \partial \mathbb{D} \rightarrow B\left(\mathcal{D}_{T}\right)$ denote the defect function of $\Theta_{T}$ defined by

$$
\Delta_{T}(z)=\left(I-\Theta_{T}(z)^{*} \Theta_{T}(z)\right)^{1 / 2} \quad \text { for a.e. } z \in \partial \mathbb{D} .
$$

Consider the Hilbert space $L^{2}\left(\mathcal{D}_{T^{*}} \oplus \mathcal{D}_{T}\right) \simeq L^{2}\left(\mathcal{D}_{T^{*}}\right) \oplus L^{2}\left(\mathcal{D}_{T}\right)$ of vector-valued functions, where the underlying measure is the normalized Lebesgue measure $m$ on the unit circle $\partial \mathbb{D}$. Therefore a function $f: \partial \mathbb{D} \rightarrow \mathcal{D}_{T^{*}} \oplus \mathcal{D}_{T}$ belongs to $L^{2}\left(\mathcal{D}_{T^{*}} \oplus \mathcal{D}_{T}\right)$ exactly if $\langle f(z) x, y\rangle$ is a Lebesgue measurable function of $z$, for every $x, y \in \mathcal{D}_{T^{*}} \oplus \mathcal{D}_{T}$, and the norm $\|f\|:=\left(\int_{\partial \mathbb{D}}\|f(z)\|^{2} d m(z)\right)^{1 / 2}$ is finite. (See [35], [12] or [14].) Let $M$ denote the unitary operator of multiplication by the identity function $\chi(z)=z$ in the Hilbert space $L^{2}\left(\mathcal{D}_{T^{*}} \oplus \mathcal{D}_{T}\right)$. Furthermore, let $H^{2}\left(\mathcal{D}_{T^{*}}\right)$ stand for the Hardy subspace of $L^{2}\left(\mathcal{D}_{T^{*}}\right)$, that is, $H^{2}\left(\mathcal{D}_{T^{*}}\right)$ consists of those functions whose Fourier coefficients of negative indices are zero. Now the model space for $T$ is defined as

$$
\mathcal{H}\left(\Theta_{T}\right):=\left[H^{2}\left(\mathcal{D}_{T^{*}}\right) \oplus\left(\Delta_{T} L^{2}\left(\mathcal{D}_{T}\right)\right)^{-}\right] \ominus\left\{\Theta_{T} w \oplus \Delta_{T} w: w \in H^{2}\left(\mathcal{D}_{T}\right)\right\},
$$

and the model operator $S\left(\Theta_{T}\right)$ is the compression of $M$ to this space:

$$
S\left(\Theta_{T}\right):=P_{\mathcal{H}\left(\Theta_{T}\right)} M \mid \mathcal{H}\left(\Theta_{T}\right) .
$$


One of the main achievements of the Sz.-Nagy-Foiaş theory of contractions is the central theorem claiming that the operator $S\left(\Theta_{T}\right)$ is unitarily equivalent to the c.n.u. contraction $T$. Conversely, starting out from an arbitrary analytic, purely contractive operator-valued function $\Theta: \mathbb{D} \rightarrow \mathcal{B}\left(\mathcal{E}, \mathcal{E}_{*}\right)$, the model operator $S(\Theta)$ constructed as above is a c.n.u. contraction. Therefore, dealing with c.n.u. contractions we can suppose in the sequel that $T$ is a model operator of the form $S(\Theta)$.

The minimal unitary dilation $U_{T}$ of $T=S(\Theta)$ coincides with the restriction of the operator $M$ of multiplication by the identity function $\chi$ to the subspace $\mathcal{K}_{T}=L^{2}\left(\mathcal{E}_{*}\right) \oplus\left(\Delta L^{2}(\mathcal{E})\right)^{-}$. Since the wandering subspace $\mathcal{L}_{T}$ is now of the form $\mathcal{L}_{T}=\{\Theta w \oplus \Delta w: w \in \mathcal{E}\}$, the space $\mathcal{R}_{*, T}$ of the $*$-residual part of $U_{T}$ has no good representation in the functional model. Hence we introduce the operator-valued function

$$
\begin{gathered}
W: \partial \mathbb{D} \rightarrow \mathcal{B}\left(\mathcal{E}_{*} \oplus \mathcal{E}\right), \\
W(z):=\left[\begin{array}{cc}
-\Delta_{*}(z) & \Theta(z) \\
\Theta(z)^{*} & \Delta(z)
\end{array}\right] \quad(z \in \partial \mathbb{D})
\end{gathered}
$$

of Julia type and the selfadjoint, unitary operator $\widehat{W} \in \mathcal{B}\left(L^{2}\left(\mathcal{E}_{*} \oplus \mathcal{E}\right)\right)$ of multiplication by $W$. Here $\Delta$ and $\Delta_{*}$ are the defect functions of $\Theta$ and $\Theta^{*}$, respectively. It is easy to see that $\widehat{W} \mathcal{L}_{T}=\mathcal{E}$, and it can be verified (see [19]) that $\widehat{W} \mathcal{R}_{*, T}=\left(\Delta_{*} L^{2}\left(\mathcal{E}_{*}\right)\right)^{-}=: \widetilde{\mathcal{R}}_{*, T}$. Denoting by $\widetilde{R}_{*, T}$ the restriction of $M$ to $\widetilde{\mathcal{R}}_{*, T}$ it is clear that the unitary transformation $W_{*}=\widehat{W} \mid \mathcal{R}_{*, T}: \mathcal{R}_{*, T} \rightarrow \widetilde{\mathcal{R}}_{*, T}$ intertwines $R_{*, T}$ and $\widetilde{R}_{*, T}$. Thus, if $\widetilde{X}_{T}$ stands for the composition $W_{*} \widehat{X}_{T} \in \mathcal{I}\left(T, \widetilde{R}_{*, T}\right)$ then, in view of Theorem 4, we obtain the following representation of the unitary asymptote of the model operator $T=S(\Theta)$.

THEOREM 5. Let $T=S(\Theta)$ be a model operator corresponding to the purely contractive analytic function $\Theta: \mathbb{D} \rightarrow \mathcal{B}\left(\mathcal{E}, \mathcal{E}_{*}\right)$. Then the pair $\left(X_{T}, T^{(\mathrm{a})}\right)$ is equivalent to $\left(\widetilde{X}_{T}, \widetilde{R}_{*, T}\right)$ where the transformations have the following simple forms:

$$
\tilde{X}_{T}(u \oplus v)=-\Delta_{*} u+\Theta v \quad \text { for } u \oplus v \in \mathcal{H}(\Theta),
$$

and

$$
\widetilde{R}_{*, T}=M \mid\left(\Delta_{*} L^{2}\left(\mathcal{E}_{*}\right)\right)^{-}
$$

We know that $\widetilde{X}_{T}$ is one-to-one if and only if the contraction $T=S(\Theta)$ is of class $C_{1}$. Moreover, in terms of $\Theta$ the condition $T \in C_{1}$. means that the function $\Theta$ is $*$-outer, that is, $\left(\Theta^{\sim} H^{2}\left(\mathcal{E}_{*}\right)\right)^{-}=H^{2}(\mathcal{E})$, where $\Theta^{\sim}(z)=\Theta(\bar{z})^{*}$ (see [35]). On the other hand, it is not hard to check (see [19]) that

$$
\operatorname{ker} \widetilde{X}_{T}^{*}=\operatorname{ker} \Theta^{*} \cap\left(L^{2}\left(\mathcal{E}_{*}\right) \ominus H^{2}\left(\mathcal{E}_{*}\right)\right),
$$

hence $\widetilde{X}_{T}$ has dense range if and only if

$$
\operatorname{ker} \Theta^{*} \cap\left(L^{2}\left(\mathcal{E}_{*}\right) \ominus H^{2}\left(\mathcal{E}_{*}\right)\right)=\{0\} .
$$


3. Reflexivity. The nice representations of the unitary asymptote and the canonical intertwining operator given in Theorem 5 enable us to show the existence of functions in the range of $\widetilde{X}_{T}$ which are separated from zero. Namely, the following lemma was proved in [23]. (For its predecessors see [20] and [36].)

LEMMA 1. Let $\Theta: \mathbb{D} \rightarrow \mathcal{B}\left(\mathcal{E}, \mathcal{E}_{*}\right)$ be a purely contractive analytic function, let $\Gamma_{*}:=\left\{z \in \partial \mathbb{D}: \Delta_{*}(z) \neq 0\right\}$ denote the support of the defect function $\Delta_{*}$ of $\Theta^{*}$, and let $\chi_{\Gamma_{*}}$ be the characteristic function of $\Gamma_{*}$. Given any number $0<\varrho<\sqrt{2} / 2$, there exist functions $u \in H^{2}\left(\mathcal{E}_{*}\right)$ and $v \in\left(\Delta L^{2}(\mathcal{E})\right)^{-}$such that

$$
\left|\|(u \oplus v)(z)\|_{\mathcal{E}_{*} \oplus \mathcal{E}}-\chi_{\Gamma_{*}}(z)\right|<\varrho \quad \text { for a.e. } z \in \partial \mathbb{D}
$$

and

$$
\frac{\sqrt{2}}{2}-\varrho<\left\|\left(-\Delta_{*} u+\Theta v\right)(z)\right\|_{\mathcal{E}_{*}}<1+\varrho \quad \text { for a.e. } z \in \Gamma_{*} .
$$

Observe that, because of the relation $-\Delta_{*} \Theta+\Theta \Delta=0$, we have $\widetilde{X}_{T} h=$ $-\Delta_{*} u+\Theta v$ for $h=P_{\mathcal{H}(\Theta)}(u \oplus v)$.

Suppose that the unitary asymptote $T^{(\mathrm{a})}$ of $T=S(\Theta)$ is nonreductive, that is, $T^{(\mathrm{a})}$ has a nonreducing invariant subspace. In terms of $\Theta$ this means that $\Delta_{*}(z) \neq 0$ for a.e. $z \in \partial \mathbb{D}$. (See [9], [12] or [28].) The preceding lemma tells us that there exists $h \in \mathcal{H}(\Theta)$ whose transform $k=\widetilde{X}_{T} h$ is separated from zero: $\|k(z)\|_{\mathcal{E}_{*}}>1 / 2$ for a.e. $z \in \partial \mathbb{D}$. Let $\mathcal{M}_{h}:=\bigvee_{n=0}^{\infty} T^{n} h$ and $\mathcal{N}_{k}:=\bigvee_{n=0}^{\infty} \chi^{n} k$ be the cyclic invariant subspaces induced by $h$ and $k$, respectively. It is easy to see that $\widetilde{R}_{*, T}\left|\mathcal{N}_{k}=M\right| \mathcal{N}_{k}$ is a simple unilateral shift and that the non-zero transformation $\widetilde{X}_{T} \mid \mathcal{M}_{h}$ belongs to the intertwining class $\mathcal{I}\left(T\left|\mathcal{M}_{h}, \widetilde{R}_{*, T}\right| \mathcal{N}_{k}\right)$. Applying a result of H. Bercovici and K. Takahashi in [5] we conclude that the set

$$
\operatorname{Alg} \operatorname{Lat}\left(T \mid \mathcal{M}_{h}\right):=\left\{Q \in \mathcal{B}\left(\mathcal{M}_{h}\right): \operatorname{Lat} Q \supset \operatorname{Lat}\left(T \mid \mathcal{M}_{h}\right)\right\}
$$

of operators leaving invariant every invariant subspace of $T \mid \mathcal{M}_{h}$ coincides with the set

$$
H^{\infty}\left(T \mid \mathcal{M}_{h}\right):=\left\{f\left(T \mid \mathcal{M}_{h}\right)=f(T) \mid \mathcal{M}_{h}: f \in H^{\infty}\right\}
$$

of functions of $T \mid \mathcal{M}_{h}$ provided by the Sz.-Nagy-Foiaş functional calculus (see [35]).

It is easy to see that small perturbations of $h$ preserve the above property of $h$. More precisely, if $x \in H^{2}\left(\mathcal{E}_{*}\right)$ and $y \in\left(\Delta L^{2}(\mathcal{E})\right)^{-}$are functions such that

$$
\operatorname{ess} \sup \left\{\|x(z)\|_{\mathcal{E}_{*}}+\|y(z)\|_{\mathcal{E}}: z \in \partial \mathbb{D}\right\}<\frac{1}{4},
$$

then $\left\|\left(\widetilde{X}_{T} h^{\prime}\right)(z)\right\|_{\mathcal{E}_{*}}>1 / 4$ a.e. on $\partial \mathbb{D}$ for $h^{\prime}=h+P_{\mathcal{H}(\Theta)}(x \oplus y)$. Since the vectors $h^{\prime}$ form a total set in $\mathcal{H}(\Theta)$, it can be shown that the global equation Alg Lat $T=H^{\infty}(T)$ is true. Since the continuity properties of the Sz.-Nagy-Foiaş calculus immediately imply that $H^{\infty}(T)$ is contained in the closure $\operatorname{Alg} T$ of all polynomials of $T$ in the weak operator topology, we infer that $\operatorname{Alg} \operatorname{Lat} T=\operatorname{Alg} T$, that is, $T$ is reflexive (see [32]). 
Thus we have obtained the following theorem, first proved in [20] under the additional assumption $T \in C_{11}$ and then in the present form in [36].

THEOREM 6. If the unitary asymptote $T^{(\mathrm{a})}$ of the c.n.u. contraction $T$ is nonreductive, then

$$
\operatorname{Alg} \operatorname{Lat} T=H^{\infty}(T)
$$

in particular, $T$ is reflexive.

Though the unitary asymptote in the nonreductive case has proved to be a useful tool, its applicability in the reductive case is not so transparent. Indeed, answering in the negative a question posed by R. Teodorescu and V. I. Vasyunin it was shown in [21] that there exists a contraction $T$ of class $C_{11}$ such that $X_{T} h$ is a cyclic vector of the unitary asymptote $T^{(\mathrm{a})}$, for every nonzero $h$. Of course, $T^{(\mathrm{a})}$ is reductive in that case. So the following questions are still open.

QUESTION 1. Does every contraction T with a nonvanishing unitary asymptote $T^{(\mathrm{a})}$ have a nontrivial invariant subspace?

By considering the $\left[\begin{array}{cc}C_{0} & * \\ 0 & C_{1}\end{array}\right]$-type triangulations of $T$ and $T^{*}$, and applying Theorem 3, Question 1 can be reduced to the following special form.

Question $1^{\prime}$. Does every contraction $T$ of class $C_{10}$ with a reductive unitary asymptote $T^{(\mathrm{a})}$ have a nontrivial invariant subspace?

It is not true that every c.n.u. contraction with a nonvanishing unitary asymptote is reflexive. Indeed, let $T_{1} \in \mathcal{B}\left(\mathcal{H}_{1}\right)$ be a c.n.u. contraction which is similar to the unitary operator $U_{1}=M \mid \chi_{\alpha} L^{2}(m)$, where $\alpha=\{z \in \partial \mathbb{D}: \operatorname{Im} z \geq 0\}$, let $T_{2} \in \mathcal{B}\left(\mathcal{H}_{2}\right)$ be a nonreflexive operator with $\left\|T_{2}\right\|<1$ (see e.g. [8]), and consider the orthogonal sum $T=T_{1} \oplus T_{2} \in \mathcal{B}\left(\mathcal{H}=\mathcal{H}_{1} \oplus \mathcal{H}_{2}\right)$. The Riesz-Dunford calculus and Runge's theorem yield that the orthogonal projections $P_{\mathcal{H}_{1}}$ and $P_{\mathcal{H}_{2}}$ belong to $\operatorname{Alg} T$. Thus Lat $T=\operatorname{Lat} T_{1} \oplus \operatorname{Lat} T_{2}$, whence $\operatorname{Alg} \operatorname{Lat} T=\operatorname{Alg} \operatorname{Lat} T_{1} \oplus \operatorname{Alg} \operatorname{Lat} T_{2}$. Since $\operatorname{Alg} T \subset \operatorname{Alg} T_{1} \oplus \operatorname{Alg} T_{2}, A \operatorname{Alg} T_{1}=\operatorname{Alg} \operatorname{Lat} T_{1}$ and $\operatorname{Alg} T_{2} \varsubsetneqq \operatorname{Alg} \operatorname{Lat} T_{2}$, we infer that $T$ is not reflexive.

Unitary operators are known to be reflexive (see [32]). On the other hand, every contraction of class $C_{11}$ is quasi-similar to its unitary asymptote. Hence we can expect a positive answer to the following question.

QUESTION 2. Is every contraction $T$ of class $C_{11}$ reflexive?

We remark that reflexivity is not a quasi-similarity invariant in general; a counterexample can be given following a construction in [15]. Furthermore, W. R. Wogen has given an example of a (noncontractive) operator which is quasisimilar to a unitary operator but is not reflexive (see [37]).

We can get nice structure theorems if we suppose that the characteristic function has a scalar multiple. Indeed, let $T=S(\Theta)$ be a model operator of class $C_{11}$ and assume that the purely contractive analytic function $\Theta: \mathbb{D} \rightarrow \mathcal{B}\left(\mathcal{E}, \mathcal{E}_{*}\right)$ has a scalar multiple $(0 \neq) \delta \in H^{\infty}$. This means that there exists a contractive 
analytic function $\Omega: \mathbb{D} \rightarrow \mathcal{B}\left(\mathcal{E}_{*}, \mathcal{E}\right)$ such that $\Omega \Theta=\delta I_{\mathcal{E}}$ and $\Theta \Omega=\delta I_{\mathcal{E}_{*}}$. It can be shown in that case that there exists a quasi-affinity $Z \in \mathcal{I}\left(T^{(a)}, T\right)$ which nicely suits $X_{T}$, namely $Z X_{T}=\delta(T)$ and $X_{T} Z=\delta\left(T^{(\mathrm{a})}\right)$. Exploiting this strong relation between $T$ and $T^{(\mathrm{a})}$ it can be proved that $T$ is reflexive and the invariant subspace lattices Lat $T$ and Lat $T^{(a)}$ are isomorphic (see [16] and [17]).

4. Spectrum. Let $T \in \mathcal{B}(\mathcal{H})$ be again an arbitrary power bounded operator. Let us examine how the spectrum $\sigma\left(T^{(\mathrm{a})}\right)$ of the unitary asymptote $T^{(\mathrm{a})}$ relates to the spectrum $\sigma(T)$ of $T$.

The existence of the algebra homomorphism $\gamma_{T}$ between the commutants $\{T\}^{\prime}$ and $\left\{T^{(\mathrm{a})}\right\}^{\prime}$, defined in Section 1, immediately implies that $\sigma\left(T^{(\mathrm{a})}\right)$ is contained in $\sigma(T)$. Furthermore, if $T$ is of class $C_{1}$., then considering Riesz subspaces and applying Theorem 2 it can be easily verified that $\sigma\left(T^{(\mathrm{a})}\right)$ intersects every closedand-open (clopen) subset $\sigma^{\prime}$ of $\sigma(T)$. Therefore the spectra of power bounded operators of class $C_{1}$. are attached to the unit circle. We remark that if the unitary operator $T^{(\mathrm{a})}$ is absolutely continuous with respect to the Lebesgue measure $m$, then $m\left(\sigma\left(T^{(\mathrm{a})}\right) \cap \sigma^{\prime}\right)>0$ for every clopen subset $\sigma^{\prime}$ of $\sigma(T)$.

By the main results of [4] and [18] this is the only constraint on the connection between the spectra of $T$ and $T^{(a)}$ even in special classes of contractions.

THEOREM 7. Let $\alpha$ be a nonempty compact subset of the unit circle $\partial \mathbb{D}$ such that $\alpha$ coincides with the closed support of the measure $\chi_{\alpha} d m$, and let $\sigma$ be a compact subset of the closed unit disc $\mathbb{D}^{-}$such that $\sigma \supset \alpha$ and $m\left(\sigma^{\prime} \cap \alpha\right)>0$ for every nonempty clopen subset $\sigma^{\prime}$ of $\sigma$. Then there exist c.n.u. contractions $T_{1}$ and $T_{2}$ of class $C_{10}$ and $C_{11}$, respectively, such that $\sigma\left(T_{1}\right)=\sigma\left(T_{2}\right)=\sigma$ and $\sigma\left(T_{1}^{(\mathrm{a})}\right)=\sigma\left(T_{2}^{(\mathrm{a})}\right)=\alpha$.

The main ingredient in the proof of this theorem is the following lemma.

Lemma 2. Let $\alpha$ be a compact set as in Theorem 7. Then, for every positive number $K$, there exist c.n.u. contractions $A_{1}$ and $A_{2}$ of class $C_{10}$ and $C_{11}$, respectively, such that $\sigma\left(A_{j}\right)=\sigma\left(A_{j}^{(\mathrm{a})}\right)=\alpha$ and $\left\|A_{j}^{-1}\right\|>K$ for $j=1,2$.

While the proof of this lemma is fairly easy in the $C_{11}$-case, it is much more difficult in the $C_{10}$-case when $A_{1}$ is carefully selected as a restriction of a weighted bilateral shift.

Having this lemma, topological considerations and an extensive use of the Sz.Nagy-Foiaş functional calculus (the spectral mapping theorem, the von Neumann inequality) yield the theorem.

Interestingly enough, as was shown in [24], the reducing essential spectrum

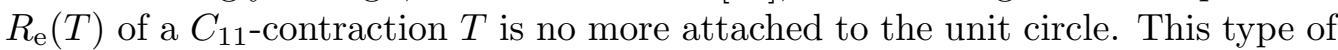
spectrum was introduced by N. Salinas in [31]; among other things, it determines the normal operators which are essentially contained in the operator $T$ as a direct summand. Namely, if $N$ is a normal operator then $T \oplus N$ is unitarily equivalent 
to $T+K$, for some compact $K$, precisely if the essential spectrum $\sigma_{\mathrm{e}}(N)$ of $N$ is contained in $R_{\mathrm{e}}(T)$.

We can see from Theorem 7 that the spectra of power bounded operators of class $C_{1}$. are the same as the spectra of contractions in the special classes $C_{10}$ and $C_{11}$. This observation leads to the following natural question.

QUESTION 3. Is every power bounded operator $T$ of class $C_{1}$. (or, in particular, of class $C_{10}$ or $C_{11}$ ) similar to a contraction?

This question, without the constraint $T \in C_{1}$., was posed by B. Sz.-Nagy and was answered in the negative by S. R. Foguel. However, the counterexamples known to the author are not of class $C_{1}$. (see [10], [11] and [26]). An affirmative answer to Question 3 would immediately extend the validity of Theorem 6 to power bounded operators.

\section{References}

[1] S. Banach, Théorie des Opérations Linéaires, Chelsea, New York, 1955.

[2] H. Bercovici, Operator Theory and Arithmetic in $H^{\infty}$, Math. Surveys Monographs 26, Amer. Math. Soc., Providence, R.I., 1988.

[3] -, Commuting power-bounded operators, Acta Sci. Math. (Szeged) 57 (1993), 55-64.

[4] H. Bercovici and L. Kérchy, On the spectra of $C_{11}$-contractions, Proc. Amer. Math. Soc. 95 (1985), 412-418.

[5] H. Bercovici and K. Takahashi, On the reflexivity of contractions on Hilbert space, J. London Math. Soc. (2) 32 (1985), 149-156.

[6] J. B. Conway, A Course in Functional Analysis, Springer, New York, 1985.

[7] M. Day, Means for bounded functions and ergodicity of the bounded representations of semigroups, Trans. Amer. Math. Soc. 69 (1950), 276-291.

[8] J. A. Deddens and P. A. Fillmore, Reflexive linear transformations, Linear Algebra Appl. 10 (1975), 89-93.

[9] N. Dunford and J. Schwartz, Linear Operators. II, Interscience, New York, 1963.

[10] S. R. Foguel, A counterexample to a problem of Sz.-Nagy, Proc. Amer. Math. Soc. 15 (1964), 788-790.

[11] P. R. Halmos, On Foguel's answer to Nagy's question, ibid., 791-793.

[12] H. Helson, Lectures on Invariant Subspaces, Academic Press, New York, 1964.

[13] E. Hewitt and K. Ross, Abstract Harmonic Analysis. I, Springer, Berlin, 1963.

[14] E. Hille and R. S. Phillips, Functional Analysis and Semi-groups, Amer. Math. Soc., Providence, 1957.

[15] T. B. Hoover, Quasi-similarity of operators, Illinois J. Math. 16 (1972), 678-686.

[16] L. Kérchy, A description of invariant subspaces of $C_{11}$-contractions, J. Operator Theory 15 (1986), 327-344.

[17] -, Contractions being weakly similar to unitaries, in: Oper. Theory: Adv. Appl. 17, Birkhäuser, Basel, 1986, 187-200.

[18] - On the spectra of contractions belonging to special classes, J. Funct. Anal. 67 (1986), 153-166.

[19] - On the residual parts of completely non-unitary contractions, Acta Math. Hungar. 50 (1987), 127-145.

[20] - Invariant subspaces of $C_{1}$--contractions with non-reductive unitary extensions, Bull. London Math. Soc. 19 (1987), 161-166. 
[21] L. Kérchy, On a conjecture of Teodorescu and Vasyunin, in: Oper. Theory: Adv. Appl. 28, Birkhäuser, Basel, 1988, 169-172.

[22] - Isometric asymptotes of power bounded operators, Indiana Univ. Math. J. 38 (1989), 173-188.

[23] - On the functional calculus of contractions with nonvanishing unitary asymptotes, Michigan Math. J. 37 (1990), 323-338.

[24] - On the reducing essential spectra of contractions, Acta Sci. Math. (Szeged) 57 (1993), 175-198.

[25] N. K. Nikolskiŭ and V. I. Vasyunin, A unified approach to function models, and the transcription problem, in: Oper. Theory: Adv. Appl. 41, Birkhäuser, Basel, 1989, 405-434.

[26] V. V. Peller, Estimates of functions of power bounded operators on Hilbert space, J. Operator Theory 7 (1982), 341-372.

[27] V. Pták, Construction of dilations, Exposition. Math. 10 (1992), 151-170.

[28] H. Radjavi and P. Rosenthal, Invariant Subspaces, Springer, New York, 1973.

[29] F. Riesz and B. Sz.-Nagy, Functional Analysis, Ungar, New York, 1955

[30] W. Rudin, Functional Analysis, McGraw-Hill, New York, 1973.

[31] N. Salinas, Reducing essential eigenvalues, Duke Math. J. 40 (1973), 561-580.

[32] D. Sarason, Invariant subspaces and unstarred operator algebras, Pacific J. Math. 17 (1966), 511-517.

[33] B. Sz.-Nagy, On uniformly bounded linear transformations in Hilbert space, Acta Sci. Math. (Szeged) 11 (1947), 152-157.

[34] -, Sur les contractions de l'espace de Hilbert, ibid. 15 (1953), 87-92.

[35] B. Sz.-Nagy and C. Foias, Harmonic Analysis of Operators on Hilbert Space, NorthHolland and Akadémiai Kiadó, Amsterdam-Budapest, 1970.

[36] K. Takahashi, The reflexivity of contractions with nonreductive $*$-residual parts, Michigan Math. J. 34 (1987), 153-159.

[37] W. R. Wogen, On reflexivity and quasisimilarity, preprint. 